\title{
Hesperidin from Citrus seed induces human hepatocellular carcinoma HepG2 cell apoptosis via both mitochondrial and death receptor pathways
}

\author{
Ratana Banjerdpongchai ${ }^{1}$ - Benjawan Wudtiwai ${ }^{1}$ - Patompong Khaw-on ${ }^{1}$. \\ Wasitta Rachakhom ${ }^{1}$ • Natthachai Duangnil ${ }^{1}$ • Prachya Kongtawelert ${ }^{1}$
}

Received: 18 May 2015 / Accepted: 7 July 2015 / Published online: 21 July 2015

(C) The Author(s) 2015. This article is published with open access at Springerlink.com

\begin{abstract}
Citrus seeds are full of phenolic compounds, such as flavonoids. The aims of this study were to identify the types of flavonoids in Citrus seed extracts, the cytotoxic effect, mode of cell death, and signaling pathway in human hepatic cancer HepG2 cells. The flavonoids contain anticancer, free radical scavenging, and antioxidant activities. Neohesperidin, hesperidin, and naringin, active flavanone glycosides, were identified in Citrus seed extract. The cytotoxic effect of three compounds was in a dose-dependent manner, and $\mathrm{IC}_{50}$ levels were determined. The sensitivity of human HepG2 cells was as follows: hesperidin $>$ naringin $>$ neohesperidin $>$ naringenin. Hesperidin induced HepG2 cells to undergo apoptosis in a dose-dependent manner as evidenced by the externalization of phosphatidylserine and determined by annexin V-fluorescein isothiocyanate and propidium iodide staining using flow cytometry. Hesperidin did not induce the generation of reactive oxygen species, which was determined by using $2^{\prime}, 7^{\prime}$ dichlorohydrofluorescein diacetate and flow cytometry
\end{abstract}

Ratana Banjerdpongchai

ratana.b@cmu.ac.th

Benjawan Wudtiwai

boaunn@gmail.com

Patompong Khaw-on

iaongstudio@gmail.com

Wasitta Rachakhom

wasitta_leejeen@live.com

Natthachai Duangnil

mk_chatchai@hotmail.com

Prachya Kongtawelert

prachya.kongtawelert@gmail.com

1 Department of Biochemistry, Faculty of Medicine, Chiang Mai University, Chiang Mai 50200, Thailand method. The number of hesperidin-treated HepG2 cells with the loss of mitochondrial transmembrane potential increased concentration dependently, using $3,3^{\prime}$ dihexyloxacarbocyanine iodide employing flow cytometry. Caspase- $9,-8$, and -3 activities were activated and increased in hesperidin-treated HepG2 cells. Bcl-xL protein was downregulated whereas Bax, Bak, and tBid protein levels were upregulated after treatment with hesperidin in a dosedependent manner. In conclusion, the bioflavanone from Citrus seeds, hesperidin, induced human HepG2 cell apoptosis via mitochondrial pathway and death receptor pathway. Citrus seed flavonoids are beneficial and can be developed as anticancer drug or food supplement, which still needs further in vivo investigation in animals and human beings.

Keywords Citrus seeds · Apoptosis · Cancer · HepG2 cells · Hesperidin · Flavonoids

\section{Introduction}

The incidence of liver cancer is in the first fifth ranks of mortality in the world. The risk and precipitating factors or causes include viral hepatitis B and C infection, alcoholic cirrhosis, non-alcoholic fatty liver related to obesity, prolonged consumption of drugs, or chronic hepatic exposure to xenobiotics such as aflatoxin B1 $[1,2]$.

Chemotherapy, immunotherapy, intravenous drug embolization, and surgery are employed to treat hepatoma cancer patients. However, the effectiveness and side effects remain a problematic issue.

Recent research on anticancer effect of natural products has been focused on cancer cell death induction. Cancer cell death induced by chemotherapy or natural products can be via apoptosis (type I programmed cell death), autophagic cell death 
(type II programmed cell death), and necroptosis (programmed necrosis) [3-5]. The apoptotic cells are characterized by cell membrane blebbing, cytosolic shrinkage, condensed nuclei, and apoptotic bodies with the externalization of phosphatidylserine to the outer layer of cell membrane, whereas for the autophagic cells by the generation of autophogosomes and autophagolysosomes in the cytoplasm with Atg gene expression and necroptotic cells by cell swelling and the loss of cell membrane integrity and inhibition by necrostatin 1 [6].

Flavonoids are abundantly found in fruits and vegetables including grains. Most Citrus species contains substantial amount of limonoids, flavonoids, and carotenoids in the forms of both glycoside or aglycone. The aglycone forms, naringenin and hesperetin, are the most important flavanones [7]. These phytochemical compounds contain antioxidant, antiproliferative, antiviral, antiallergic, antiinflammatory, antiatherosclerotic, and anticancer activities [8].

The structure of glycosides determines the taste of Citrus, for example, the compounds consist of rhamnosyl-alpha-1,2-glucose or disaccharide residue (ramnosyl-alphai-1,6 glucose) give the sweet taste, whereas rutinosides and neohesperidosides provide bitter taste [9].

Hesperidin is an active flavanone glycoside found in the Citrus juice, whole fruit, and peel. Some glycosids are more toxic to cancer cells, and some are less than their aglycone derivatives, depending on the types of flavonoids [10]. Citrus bioactive compounds also induce significantly phase II detoxifying enzyme activities, such as quinone reductase and glutathione S-transferase [11]. Flavanones, hesperidin, narirutin, and didymin, are present in orange and lemon juices [9].

Citrus flavonoids such as limonin and its glucosides have been reported to inhibit colon adenocarcinoma SW480 cell proliferation through apoptosis via intrinsic pathway [12]. In a comparative study of limonoid effect on cancer cell cytotoxicity, both aglycones and glycosides of limoinoids possess strong antiproliferative activity, but the latter are the apoptosis-inducing forms in neuroblastoma SH-SY5Y cell line [13].

Since, Citrus peel and seed are also composed of polyphenolic derivatives, such as phenolic acids and flavonoids. Citrus peel contains more flavonoids than the seed [14]. The Citrus peel and seed are by-products. It is necessary to measure their bioflavonoids' contents and activities on cancer cell growth and the mechanism involved. There has never been any study on the amount and types of flavonoids in Citrus seed and the effect of Citrus flavonoids on human hepatic cancer cell proliferation; hence, the aims of this investigation were to identify the types of active compounds in Citrus seed and the inhibitory effect of Citrus seed flavonoids on human hepatocellular cancer HepG2 cell growth together with mechanism of cell death.

\section{Materials and methods}

\section{Reagents}

Bioflavonoids and ethanolic crude extract from Citrus seeds were obtained from Ruiheng Industry Co., Limited., Hefei City, China. Dimethyl sulfoxide (DMSO), 3-(4,5 dimethylthiazol-2-yl)-2,5-diphenyltetrazolium bromide (MTT dye), 2', 7'-dichlorohydrofluorescein diacetate (DCFH-DA), neohesperidin, hesperidin, naringin, and naringenin were obtained from Sigma-Aldrich, St. Louis, MO, USA. Dulbecco's modified Eagle medium (DMEM), fetal bovine serum, penicillin $\mathrm{G}$ sodiumm and streptomycin were from GibcoBRL, Invitrogen Life Science Technologies, Thermo Fisher Scientific Inc., Waltham, MA, USA. Annexin V-Fluos kit and complete mini-protease inhibitor cocktail were obtained from Roche, Indianapolis, IN, USA. Caspase determining kits were obtained from Invitrogen Life Science Technologies, Thermo Fisher Scientific Inc., Waltham, MA, USA. Mouse monoclonal antibody to $\mathrm{Bcl}-2$ associated $\mathrm{X}$ protein (Bax) and rabbit polyclonal to $\mathrm{Bcl}-\mathrm{xL}$, Bak, and Bid, and horseradish peroxidase (HRP)-conjugated secondary antibodies were obtained from Abcam, Cambridge, UK. Super Signal West Pico Chemiluminescent Substrate was obtained from Thermo Fisher Scientific Inc. Waltham, MA, USA.

\section{Characterization of active compounds in bioflavonoids from Citrus seeds}

The commercial available bioflavonoids from Citrus seeds were separated through high-performance liquid chromatography by using HP (Hewlett-Packard, CA, USA) 1050 gradient liquid chromatography with DAD $1050 \mathrm{M}$ coupled to a Chemstation HP and a reverse phase column Luna C18 $5 \mu \mathrm{m} \times 150 \times 2.1 \mathrm{~mm}$ (Phenomenex, Torrance, CA, USA). The running solvents were as follows: $0.1 \%$ formic acid in water and $0.1 \%$ formic acid in acetonitrile. Gradients were as follows: 0-35 min 6-50\%, 35-45 min 50-100\% and then back to $6 \%$. The column was equilibrated for $15 \mathrm{~min}$ prior to analysis. The flow rate was $0.4 \mathrm{ml} / \mathrm{min}$, injection volume was $10 \mu \mathrm{l}$, and column temperature was at $40{ }^{\circ} \mathrm{C}$. The UV-Vis spectra were recorded from 280 to $400 \mathrm{~nm}$, with detection at 280 and $365 \mathrm{~nm}$.

\section{Cell culture}

Human hepatocellular carcinoma HepG2 cell line was kindly given by Associate Professor Dr. Prachya Kongtawelert, Excellence Center of Tissue Engineering and Stem Cells, Department of Biochemistry, Faculty of Medicine, Chiang Mai University. HepG2 cells were cultured in DMEM medium supplemented with $10 \%$ fetal bovine serum with $25 \mathrm{mM}$ sodium 
bicarbonate, $20 \mathrm{mM}$ HEPES, $100 \mathrm{U} / \mathrm{ml}$ penicillin $\mathrm{G}$ sodium, and $100 \mu \mathrm{g} / \mathrm{ml}$ streptomycin at $37{ }^{\circ} \mathrm{C}$ and $5 \% \mathrm{CO}_{2}$.

\section{MTT assay}

The HepG2 cells were treated with crude ethanolic extract from Citrus seeds, bioflavonoids, and commercially available active compounds, viz., neohesperidin, hesperidin, naringin, and naringenin at various concentrations for $24 \mathrm{~h}$. Then, MTT dye (dissolved in DMSO less than $0.1 \%$ ) was added at final concentrations of $100 \mu \mathrm{g} / \mathrm{ml}$ and incubated for $4 \mathrm{~h}$ at $37^{\circ} \mathrm{C}$ in $\mathrm{CO}_{2}$ incubator. The medium was removed, and the violet crystal was dissolved in DMSO for $30 \mathrm{~min}$. The absorbance was determined by using spectrophotometer at $540 \mathrm{~nm}$ and reference wavelength at $630 \mathrm{~nm}$ (BioTek, Winooski, VT, USA). The concentrations at 10,20 , and $50 \%$ inhibitory effect on proliferation of the extract, bioflavonoid, and each active compound on human hepatocellular carcinoma cells were determined by plotting the graph of cell viability and concentrations of the natural products [15].

\section{Annexin V-FITC and PI staining and flow cytometry}

HepG2 cells were treated with hesperidin at $\mathrm{IC}_{0}, \mathrm{IC}_{10}, \mathrm{IC}_{20}$, and $\mathrm{IC}_{50}$ for $24 \mathrm{~h}$ and then stained with annexin $\mathrm{V}$-fluorescein isothiocyanate (FITC) and propidium iodide (PI) for $15 \mathrm{~min}$. The cells were washed with phosphate-buffered saline and resuspended in annexin V-FICTC and PI as indicated in the annexin Fluos kit. The stained cells were measured by using flow cytometer and analyzed with Cell Quest (software program) of flow cytometer (Becton Dickinson, Frankin Lakes, NJ, USA) [16].

\section{Determination of caspase- $9,-8$, and $-\mathbf{3}$ activities}

After, HepG2 cells were treated with hesperidin at various concentrations for $24 \mathrm{~h}$. The cells were harvested, and cell lysate was incubated with caspase-specific tetrapeptide substrates, which were labeled with $p$-nitroaniline. For caspase-9, LEHD- $p$ NA, caspase- 8 , IETD- $p$ NA and caspase-3, DEVD$p$ NA were used accordingly for measuring the activities of the corresponding caspases as described in each kit. The lysate was incubated with each substrate for $1 \mathrm{~h}$, and then, the absorbance was determined by spectrophotometry technique (using microplate reader, BioTek, Winooski, VT, USA) [17].

\section{MTP assay by flow cytometry}

Twenty-four-hour hesperidin-treated hepatoma HepG2 cells were incubated with dihexyloxacarbocyanine iodide $\left(\mathrm{DiOC}_{6}\right)$ at $40 \mathrm{nM}$ (as final concentration) for $15 \mathrm{~min}$ and determined for the loss of mitochondrial transmembrane potential (MTP) by using fluorescence activated cell sorter (FACS) (Becton Dickinson, Frankin Lakes, NJ, USA) [3].

\section{Determination of ROS generation}

After, the hepatic cancer HepG2 cells were incubated with hesperidin at various concentrations for $4 \mathrm{~h}$. DCFH-DA was added to the final concentration of $5 \mu \mathrm{M}$ and incubated for 15 min before determining the fluorescence intensity of DCF employing flow cytometer (Becton Dickinson, Frankin Lakes, NJ, USA) [18].

\section{Determination of proteins involved in apoptosis pathway by Western blot}

HepG2 cells were treated with hesperidin at various concentrations for $24 \mathrm{~h}$ and washed once in ice-cold PBS. The treated cells were incubated at $4{ }^{\circ} \mathrm{C}$ for $10 \mathrm{~min}$ with ice-cold cell lysis buffer $(250 \mathrm{mM}$ sucrose, $70 \mathrm{mM} \mathrm{KCl}, 0.25 \%$ Triton X-100 in phosphate-buffered saline containing complete mini-protease inhibitor cocktail). The cell lysate was centrifuged at 20,000× $g$ for $20 \mathrm{~min}$; the supernatant $(50 \mu \mathrm{g}$, determined by Bradford method) was separated by $15 \%$ SDS-PAGE and transferred onto nitrocellulose membrane. After treating with $5 \%$ non-fat milk in PBS containing $0.2 \%$ Tween-20, membrane was incubated with mouse monoclonal antibody to Bax and rabbit polyclonal to Bcl-xL, Bak, and Bid, followed by appropriate horseradish peroxidase (HRP)-conjugated secondary antibodies $(1: 20,000)$. Protein bands were visualized on X-ray film with Super Signal West Pico Chemiluminescent Substrate. The band density was determined by using densitometer compared to the control protein actin [4].

\section{Statistical analysis}

The data were demonstrated as mean \pm S.D. performed in triplicate and three times independently. The statistical difference was determined by using one-way ANOVA, and two groups of data were compared by Student's $t$ test with the $p$ value of $<0.05$ by using SPSS program version 16.0.

\section{Results}

\section{Active compounds in Citrus seed are neohesperidin, naringin, and hesperidin}

Bioflavonoids from Citrus seed composed of several active phytochemicals, such as neohesperidin, naringin, and hesperidin. The structures of active compounds are shown in Fig. 1, viz. neohesperidin (1a), hesperidin (1b), aglycone naringenin (1c) and compared to the glycoside form of naringenin, naringin (1d). Each compound peak in the extract (striped 
Fig. 1 Chemical structure of active phytochemicals found in Citrus seed: a neohesperidin, b hesperidin, $\mathbf{c}$ naringenin, and $\mathbf{d}$ naringin
A

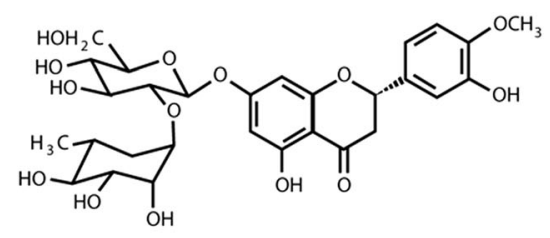

B<smiles>COc1ccc(C2CC(=O)c3c(O)cc(OC(O)C(O)COC4OC(C)(C(C)O)C(O)C(O)C4O)cc3O2)cc1O</smiles>

C<smiles>O=C1CC(c2ccc(O)cc2)Oc2cc(O)cc(O)c21</smiles>

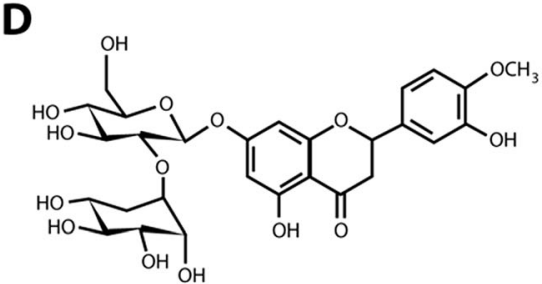

line) was compared to the standard compound, shown in solid line (Fig. 2). The standard and the active phytochemicals in Citrus seed superimposed to each other; the result was comparable to the flavanones [19]. The yields of neohesperidin, hesperidin, and naringin, identified in Citrus seed extract, were $17.11,7.69$, and $20.30 \%(w / w)$, respectively. The structures of neohesperidin and hesperidin are isomers.

\section{Cytotoxic effect of Citrus extract, bioflavonoids, and active compounds on human HepG2 cells}

Both ethanolic crude extract of Citrus seed and bioflavonoids from Citrus seed slightly inhibited HepG2 cell proliferation with the $\mathrm{IC}_{50}$ values of more than $200 \mathrm{mg} / \mu \mathrm{l}$ as shown in
Fig. 3. Bioflavonoids were toxic to HepG2 cells more than the crude extract of Citrus seed.

Hesperidin was toxic to human hepatocellular carcinoma HepG2 cells, with the $\mathrm{IC}_{10}, \mathrm{IC}_{20}$, and $\mathrm{IC}_{50}$ levels of $23.65 \pm$ $2.75,49.06 \pm 4.06$, and $150.43 \pm 12.32 \mu \mathrm{M}$, respectively. When compared to the other two bioflavonoids found in Citrus seeds, neohesperidin and naringin, both were less toxic to HepG2 cells with $\mathrm{IC}_{50}$ levels more than 200 and $172.00 \pm$ $10.39 \mu \mathrm{M}$, respectively, as shown in Fig. 4.

From the present data, flavonoids from Citrus seed inhibited human hepatic cancer HepG2 cell growth with the sensitivity as follows: hesperidin $>$ naringin $>$ neohesperidin $>$ naringenin (Fig. 4). The cytotoxicity of naringin in its glycoside form was compared with aglycone naringenin. Glycoside form was more toxic to hepatic cancer
Fig. 2 Chromatogram of the active compounds separated by high-performance liquid chromatography (HPLC) technique. The active standard phytochemicals of the flavonoids (continuous line) were compared to the compounds found in bioflavonoid from Citrus seed (striped line), viz., neohesperidin, hesperidin, and naringin

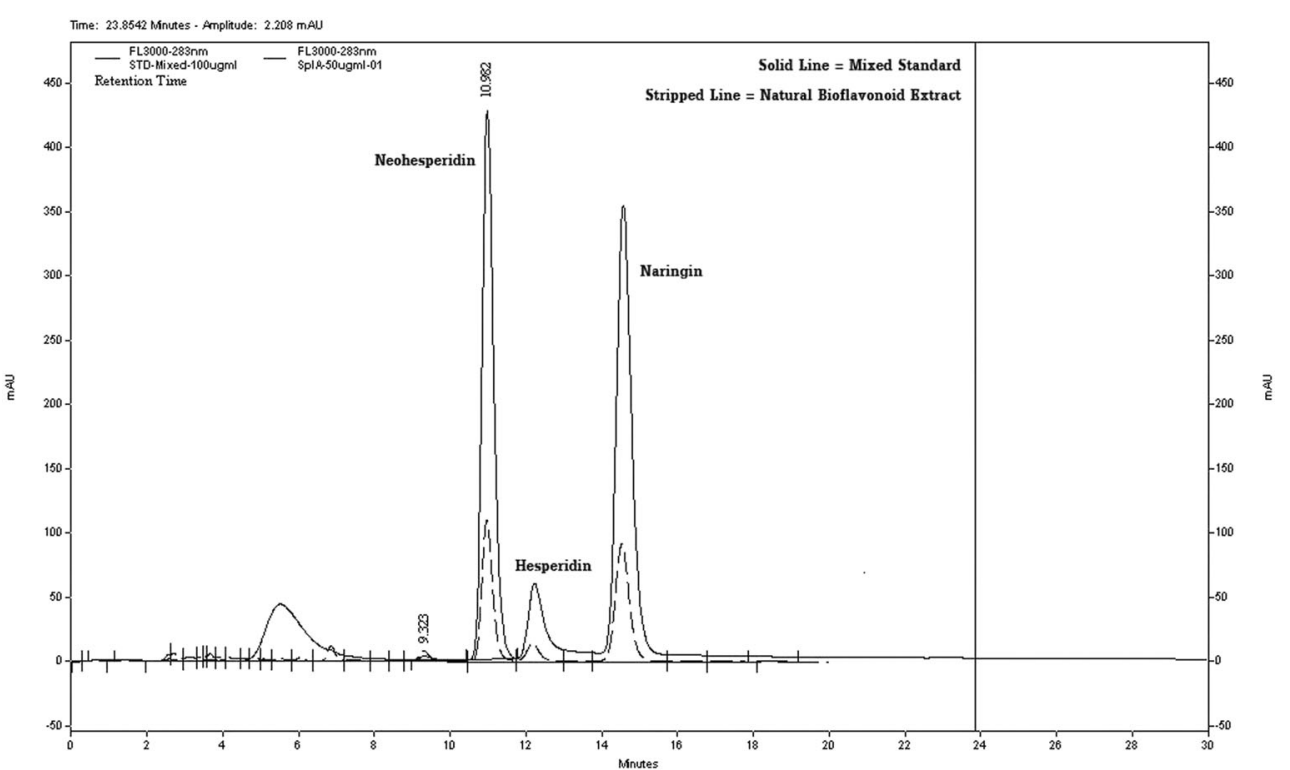




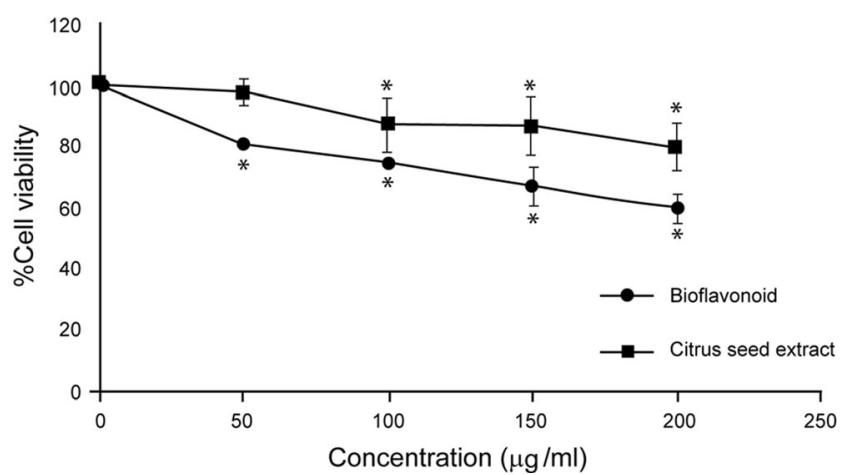

Fig. 3 Cell viability of human hepatocellular carcinoma HepG2 cell treated with crude ethanolic extract and bioflavonoids from Citrus seed. The significance of statistical value compared to control (without treatment) is marked with asterisk, $p<0.05$

cells than aglycone form in this HepG2 cell model. The most toxic compound for HepG2 cell line was hesperidin; hence, hesperidin was selected for further study of the mechanism of cell death.

\section{Hesperidin-induced apoptosis in HepG2 cells}

Determination of phosphatidylserine (PS) externalization is used to determine apoptotic or necrotic cell death by flow cytometry technique [20]. HepG2 cells treated with hesperidin at $\mathrm{IC}_{10}, \mathrm{IC}_{20}$, and $\mathrm{IC}_{50}$ concentrations showed the characteristics of apoptosis, i.e., the translocation of phosphatidylserine (PS) to the outer layer of cell membrane demonstrated by annexin V-fluorescein isothiocyanate (FITC) and propidium iodide (PI) staining at PS and DNA (nucleus), respectively, employing flow cytometry technique as shown in Fig. 5a, b. Percentage of cells in right lower quadrant (early apoptotic cells) was higher in a dose-response manner as shown in dot plot (Fig. 5a) and bar graphs (5b). The flip-flop of PS to the outer layer of cell membrane is a unique character of early apoptotic cells.

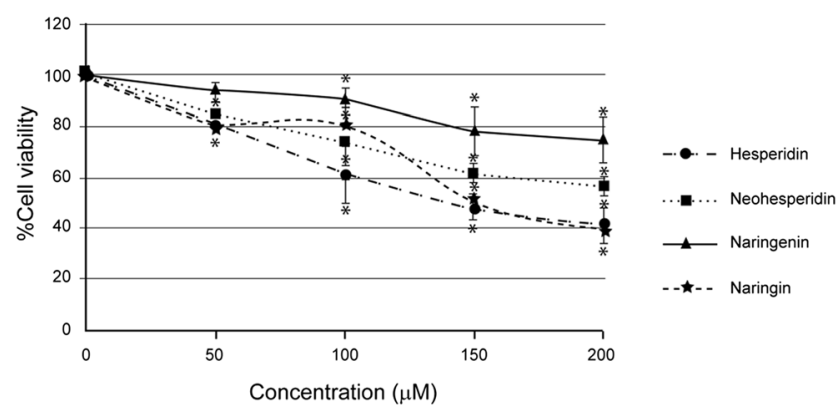

Fig. 4 Cell cytotoxicity of human hepatocellular carcinoma HepG2 cells treated with phytochemical neohesperidin, hesperidin naringin, and naringenin. HepG2 cells were treated with various concentrations of the active compounds from Citrus seed for $24 \mathrm{~h}$, and the cell viability was measured by MTT assay as described in "Materials and methods." The significance of statistical value compared to control (without treatment) is marked with asterisk, $p<0.05$

\section{Activation of caspase- $9,-8$, and -3 activities in hesperidin-induced HepG2 cell apoptosis}

Eupatorin, a naturally occurring flavone, inhibits cell proliferation in human leukemic HL-60, U937, and Molt-3 cells via inducing cell cycle arrest at G2-M phase and induces apoptotic cell death involving activation of multiple caspases via caspase-9, -8, -6, and -3/7 [21]. Therefore, HepG2 cells were treated with hesperidin at various concentrations for $24 \mathrm{~h}$, and the cells were investigated for caspase- $9,-8$, and -3 activities by using specific tetrapeptide substrates labeled with colorimetric substrates. It was found that para-nitroaniline labeling to Leu-Glu-His-Asp (LEHD- $p$ NA), which was specific for caspase-9; or Iso-Glu-Thr-Asp- $p$ NA for caspase-8; or AspGlu-Val-Asp (DEVD- $p$ NA) for caspase-3/7, respectively, is measured for the absorbance by spectrophotometer. When the tetrapeptide was cleaved accordingly after aspartate residue in each tetrapeptide, the $p$-NA is freely released and it absorbs the visible light at $410 \mathrm{~nm}$. It was found that caspase- $9,-8$, and -3 activities increased in hesperidin-treated HepG2 cells as shown in Fig. 6.

\section{Loss of MTP in hesperidin-treated HepG2 cells}

Mitochondrial pathway involves the reduction of transmembrane potential of apoptotic cancer cells induced by stigmalactam, a natural product in an aristolactam type alkaloid group [22]. In 24-h hesperidin-incubated HepG2 cells, the changes of MTP were determined by using ionic fluorochrome, 3,3'-dihexyloxacarbocyanine iodide $\left(\mathrm{DiOC}_{6}\right)$, which is up taken by the mitochondria. The relative $\mathrm{DiOC}_{6}$ fluorescence level is high in normal cells. But in apoptotic cells, the mitochondrial transmembrane permeability is lost and allowed the dye to leak into cytoplasm leading to less fluorescence intensity (Fig. 7a). Hesperidin caused the reduction of MTP, and percentage of cells with decreased fluorescence intensity was significantly increased dose dependently as shown in Fig. $7 b$.

\section{The absence of reactive oxygen species generation in hesperidin-treated HepG2 cells}

Apoptosis pathway via intrinsic and extrinsic pathways can induce the ROS generation via mitochondria and blocking NF-kappaB transcription factor in non-small cell lung cancer A549 cells induced by a histone deacetylase (HDAC) inhibitor, romidepsin [23, 24]. Human hepatocellular carcinoma HepG2 cells were treated with hesperidin for $4 \mathrm{~h}$ and stained with 2',7'-dichlorohydrofluorescein diacetate (DCFH-DA) and using flow cytometer to detect the fluorescence intensity of $2^{\prime}, 7^{\prime}$-dichlorofluorescein (DCF). The ester bond in DCFHDA is cleaved by esterase in cytoplasm to obtain DCFH, and then, DCFH is oxidized by peroxide radicals or hydrogen 
Fig. 5 Apoptotic cell death induced by hesperidin. The apoptotic HepG2 cells were stained with annexin V-

fluorescein isothiocyanate and propidium iodide and employing flow cytometry technique as described in "Materials and methods." The dot plots (a) of cells in each concentration of hesperidin are (a) control (without hesperidin treatment) and various concentrations of hesperidin at $(b)$ $\mathrm{IC}_{10},(c) \mathrm{IC}_{20}$, and (d) $\mathrm{IC}_{50}$ in $\mu \mathrm{M}$ unit. Bar graphs (b) of the percentage of early apoptotic cells in each concentration are shown. The significance of statistical value compared to control (without treatment) is marked with asterisk, $p<0.05$

A

a.

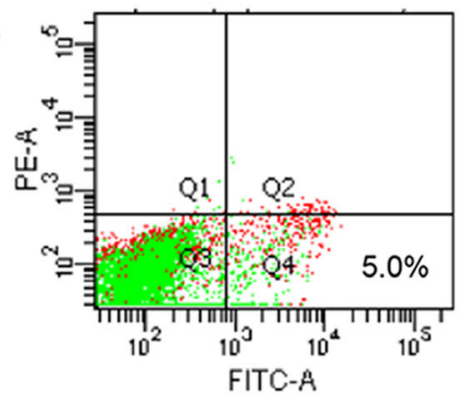

C.

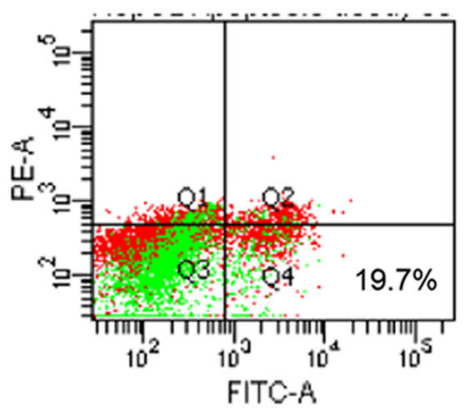

b.

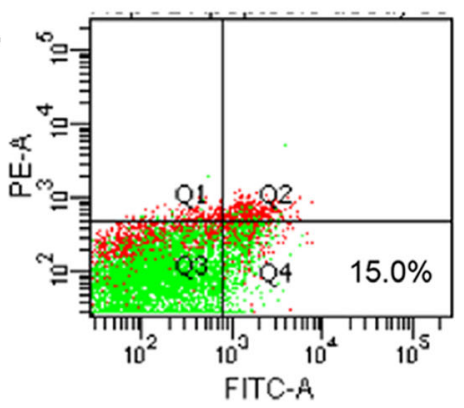

d.

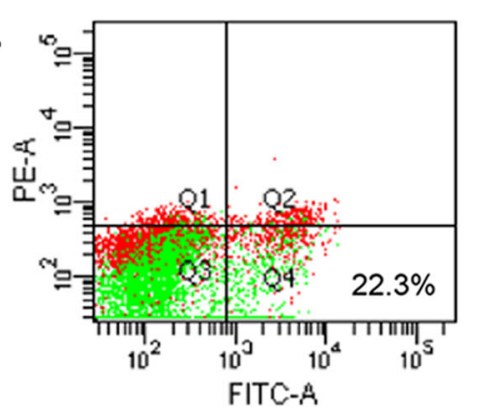

B
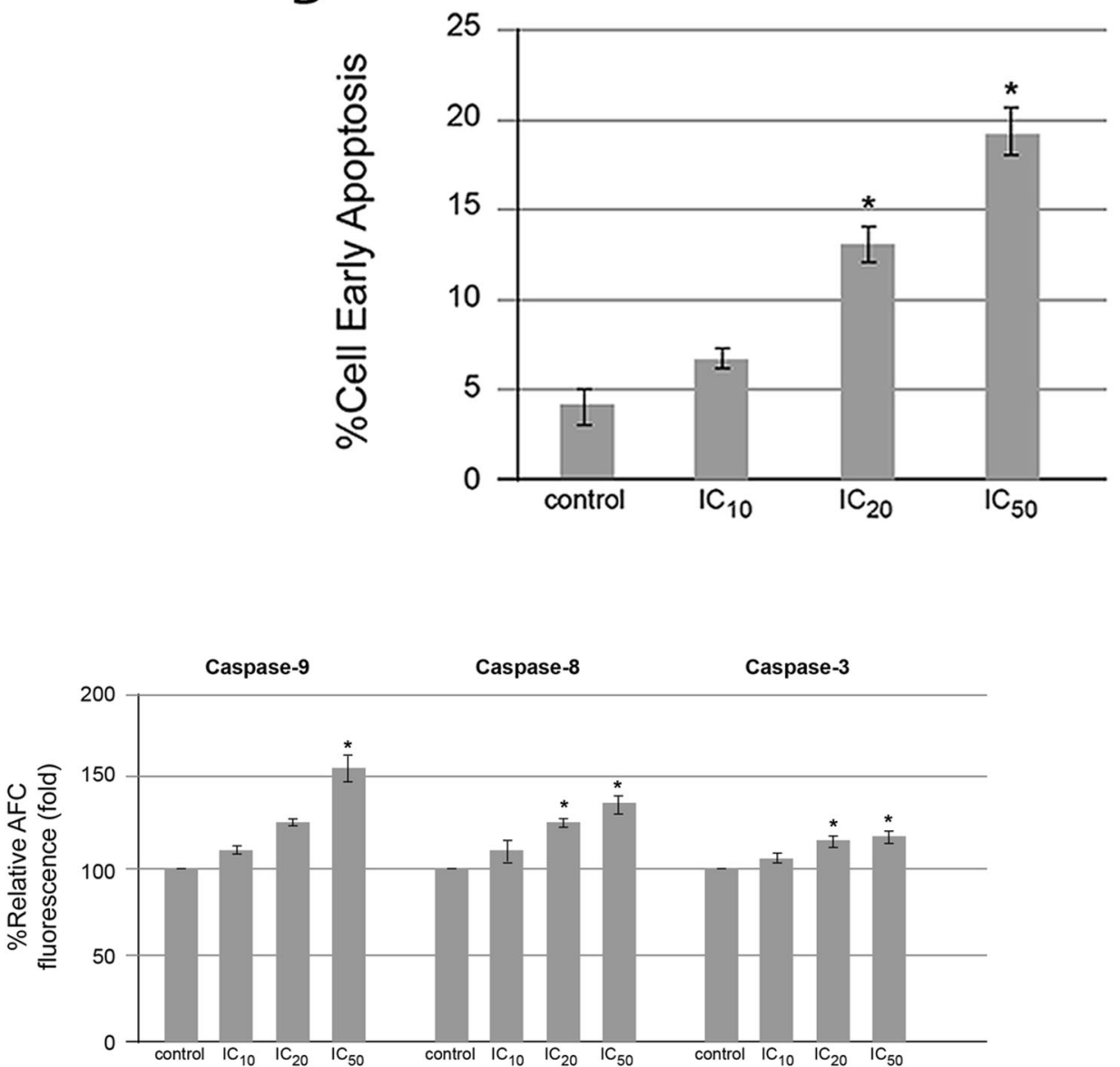

Fig. 6 Caspase activities of hesperidin-treated HepG2 cells. The caspase$9,-8$, and -3 activities of HepG2 cells treated with hesperidin for $24 \mathrm{~h}$ were measured by using specific tetrapeptide substrates tagged with para- nitroaniline, and the absorbance of cleaved $p$-NA was determined by spectrophotometer. The significance of statistical value compared to control (without treatment) is marked with asterisk, $p<0.05$ 
Fig. 7 Reduction of mitochondrial transmembrane potential (MTP) of HepG2 cells after treatment with hesperidin. The human hepatic cancer cells were treated with hesperidin at $\mathrm{IC}_{0}, \mathrm{IC}_{10}, \mathrm{IC}_{20}$, and $\mathrm{IC}_{50}$ concentrations for $24 \mathrm{~h}$, and MTP was measured by staining with $\mathrm{DiOC}_{6}$ employing flow cytometry. The relative fluorescence intensity was measured. The histograms (a) and bar graphs (b) of percentage of cells with loss of MTP are shown. The significance of statistical value compared to control (without treatment) is marked with asterisk, $p<0.05$
A a.

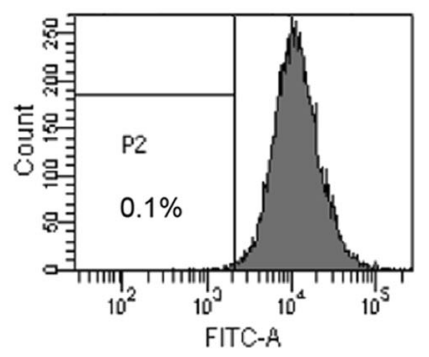

C.

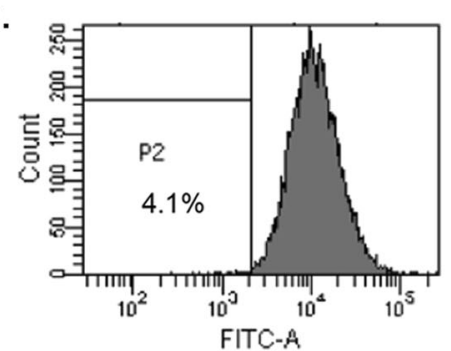

b.

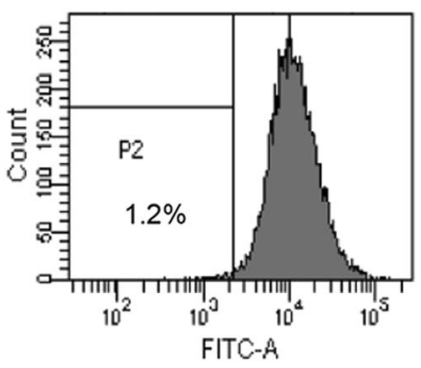

d.

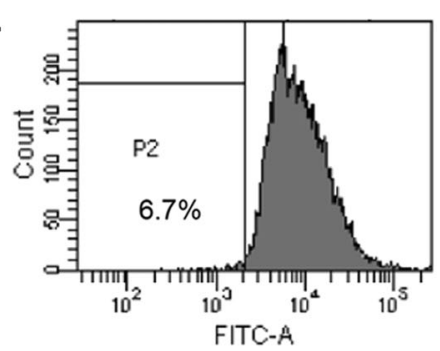

B

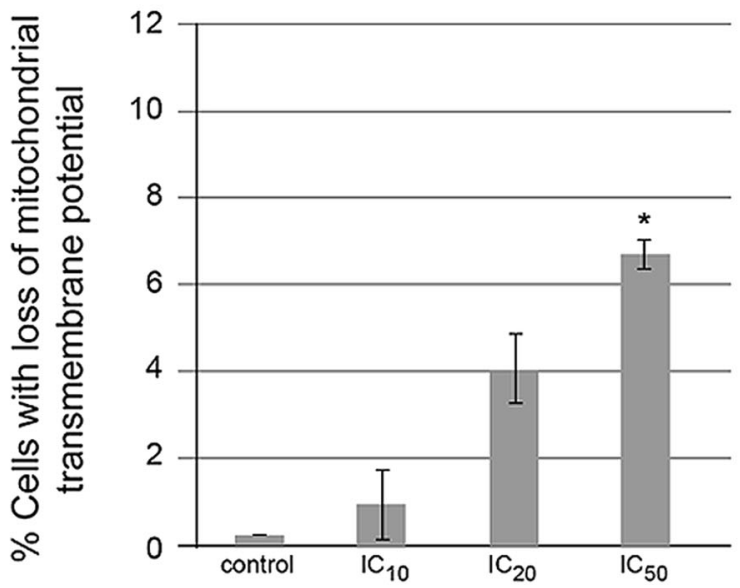

peroxide to yield DCF giving green fluorescence. By employing flow cytometry technique, the relative fluorescence intensity was compared with that of control (without treatment). There was no statistically significant alteration of the ROS generation when compared to the control (without hesperidin treatment), $p$ value $>0.05$ (Fig. 8).

\section{Increased levels of proapoptotic Bcl-2 family proteins and a decrease in antiapoptotic Bcl-xL protein}

The B-cell lymphoma 2 (Bcl-2)/Bax ratio is used as a measure of vulnerability to apoptosis [25]. The $\mathrm{Bcl}-\mathrm{xL} / \mathrm{Bax}$ was applied in the same aspect for determining the apoptosis of HepG2 cells when incubated with hesperidin, a flavonoid, found in Citrus seeds. Protein expression levels of the Bax 
Fig. 9 Apoptotic Bcl-2 family protein expression levels in hesperidin-treated HepG2 cells. HepG2 cells were treated with hesperidin at various concentrations for $24 \mathrm{~h}$. The expression levels of proapoptotic Bax (a), antiapoptotic Bcl-xL (b) and proapoptotic Bak and $\mathrm{BH}$-only $\mathrm{Bcl}-2$ family protein $\mathrm{Bid}$ together with truncated form (tBid) (c) were demonstrated by Western blot. The number under the bands was the fold (s) compared to control as verified by using actin as a constitutive protein, whereas the number above Bid bands was its expression levels compared to actin
A

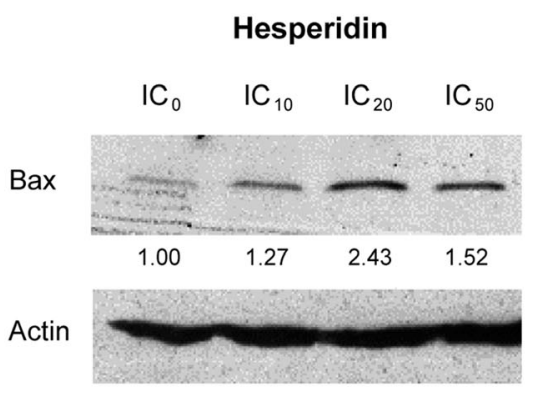

B

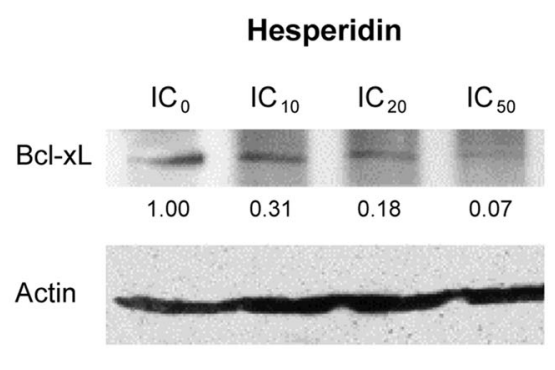

C

Hesperidin

$\begin{array}{llll}I C_{0} & I C_{10} & I C_{20} & I C_{50}\end{array}$

Bak

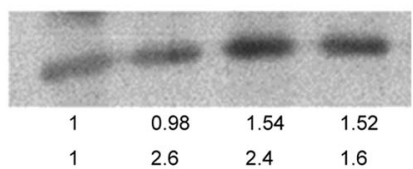

Bid

tBid

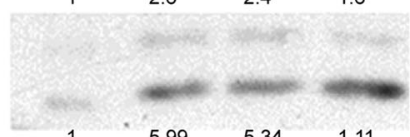

Actin

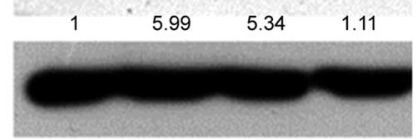

(Fig. 9a), Bcl-xL (Fig. 9b), and Bak, Bid, and tBid proteins (Fig. 9c) were measured by using immunoblot. The expression of proapoptotic Bax, Bak, and tBid proteins was higher whereas the Bcl-xL and proform of Bid protein levels were lower (compared to untreated condition) in a dose-response manner (Fig. 9). Truncated Bid (tBid) is a marker of caspase- 8 activity in proteolysis of Bid, a BH3-only protein in Bcl-2 family.

\section{Discussion}

Citrus seed contains valuable bioflavonoids with the bioactivities on apoptosis induction in human hepatocellular cancer cells in an in vitro model. The source of Citrus bioflavonoid in the present experiment was from the Chinese Company. The active flavanone glycosides were neohesperidin, hesperidin, and naringin, of which the structures are shown in Fig. 1 and their HPLC chromatogram in Fig. 2. Even though the composition of Citrus flavonoids in different species and cultivars of Citrus fruits grown in different areas produces different types of flavonoids, the present report of three flavanone glycosides corresponded to the same three flavanones found in Citrus juice, or whole fresh fruit, or pulp, or peel, or albedo and Citrus flavonoid contents in traditional Chinese medicinal (TCM) food ingredients of Eastern China, such as from Citrus aurantium L. [19, 26]. The structure of these three compounds are identified by employing high-performance liquid chromatography (HPLC) and gas chromatography-mass spectrometry (GC-MS) techniques [26].

The cytotoxic effect of hesperidin was highest (Fig. 4), and this phytochemical was selected for further experiments to identify mode and mechanism of cell death. Hesperidin can induce both apoptotic and autophagic cell death in colon carcinogenesis [27]. There are also reports demonstrating that green tea polyphenols [5] and shikonin [28] can induce cancer cell death via necroptosis, which is a regulated or programmed necrosis. Therefore, the mode of cell death induced by Citrus flavanone, hesperidin, was identified in hepatic cancer cells. It was found that the HepG2 cell death was early apoptosis in a dose-response manner because of the externalization of phosphatidylserine without propidium iodide staining to the DNA, which was detected by annexin V-FITC/PI and flow cytometry (Fig. 5a, b).

Since the apoptotic cell death can be activated through intrinsic (mitochondrial) and/or extrinsic (death receptor) pathways. The initiator caspase- 9 involves mitochondrial pathway whereas caspase- 8 is mediated in death receptor pathway. Caspase-3/6/7 are effector or executioner caspases, and they are activated in both pathways, and these caspase activities were measured to confirm the apoptotic cell death [29]. The molecular targets of hesperidin are caspases, Bcl-2, and Bax for the induction of apoptosis and cyclooxygenase-2 (COX-2), matrix metalloproteinase-2 (MMP-2), and MMP-9 for the inhibition of angiogenesis and metastasis [30]. Hesperidin induces human colon cancer SNU-C4 cell apoptosis as 
determined by a decrease in messenger RNA (mRNA) expression of $b c l-2$ and an increase of bax mRNA levels with an increase of caspase- 3 expression and activity [31]. Hence, the caspase-9, -8 , and -3 activities and the Bcl-2 family protein levels were determined. The caspase- $9,-8$, and -3 activities were significantly induced and increased in hesperidin-treated cells in a dose-response manner and especially at $\mathrm{IC}_{50}$ level (Fig. 6). Percentage of HepG2 cells with the loss of MTP was significantly increased at $\mathrm{IC}_{50}$ level whereas the mean fluorescence intensity of $\mathrm{DiOC}_{6}$ decreased. This suggested the involvement of mitochondrial dysfunction (Fig. 7).

ROS levels in the HepG2 cells after treatment with hesperidin did not change (Fig. 8) even though the increased ROS production is evidenced in some natural product-inducing cancer cell apoptosis [3]. This might be as hesperidin did not induce apoptosis via oxidative stress or/and its antioxidant/ prooxidant activities are balanced resulting in an unchanging of the DCF fluorescence intensity in the hesperidin-treated cells. Nevertheless, the ROS may not be the peroxide species since DCFH detects only hydrogen peroxide or peroxide radicals. Other ROS such as superoxide anion radicals, hydroxyl radicals, or reactive nitrogen species (RNS) including peroxynitrite radicals may be produced.

The levels of proapoptotic proteins (Bax, Bak, and $\mathrm{tBid}$ ) were increased whereas Bcl-xL protein expression was reduced (Fig. 9), which confirmed the involvement of mitochondrial pathway. Bax and Bak translocate from cytoplasm to mitochondrial outer membrane forming channel for protein release from mitochondrial intermembranous space, such as cytochrome c, Smac/Diablo, and apoptosis inducing factor (AIF) [32] to mediate apoptosis characters, which are formation of apoptosome, DNA degradation, and activation of caspase-9 and 3. Caspase-8-activated proteolysis of Bid can be mediated from the death receptor to mitochondrial dysfunction pathway as demonstrated by an increase in truncated Bid (tBid) level (Fig. 9). This confirmed the mechanism of apoptotic cell death to be via both mitochondrial and death receptor apoptotic pathways.

The anticancer potential of hesperidin is demonstrated by inhibiting cancer cell growth and proliferation via apoptosis induction [30]. The Citrus hesperidin is also reported to induce apoptosis in human hematopoietic pre-B NALM-6 leukemic cells by inducing p53 and inhibiting NF-kappaB signaling pathways [33].

In addition, hesperidin can inhibit human Burkitt's lymphoma Ramos cell proliferation and induce apoptosis by abrogating the constitutive and doxorubicin (Dox)-induced NFkappaB activation [34]. The potential strategy using Citrus bioflavonoids with the conventional heterocyclic drugs has been proposed to treat invasive brain cancer (gliomas) [35]. Hesperidin increases cytotoxicity of Dox on human cervical cancer HeLa cells [36], but in Dox-sensitive cancer cells, there is no synergistic or additive effect of hesperidin on apoptotic induction [37]. This phytochemical can attenuate cisplatininduced acute renal injury by reducing oxidative stress, inflammation, and DNA damage, which are the adverse effects of this drug through ROS production and apoptosis in rat kidneys. Therefore, the benefit of combining hesperidin with other chemotherapeutic agents remains controversial, which may depend on the types of drugs and cancer cells.

There are many reports of clinical targeted strategy against Bcl-2 family proteins for cancer therapy. A small-molecule $\mathrm{Bcl}-2-\mathrm{BH} 4$ antagonist is highly selective to the antiapoptotic Bcl-2 family proteins with specific binding affinity, changing their conformation and converting the antiapoptotic proteins to cell death inducers. The compound suppresses the growth of human cancer xenografts in mice [38]. Another type of small-molecule, Bax agonist, also induces conformational alteration in Bax protein by blocking serine 184 phosphorylation, enhancing the formation of Bax oligomers to become the channels at mitochondrial outer membrane leading to cytochrome $\mathrm{c}$ release and apoptosis induction in human lung cancer cells [39].

Niclosamide $\left(\mathrm{C}_{13} \mathrm{H}_{8} \mathrm{C}_{12} \mathrm{~N}_{2} \mathrm{O}_{4}\right)$ is also a small-molecule drug with teniacidal anthelmintic activity against tapeworms; the mechanism of death induction is via uncoupling oxidative phosphorylation in the parasite. It is a potent STAT3 inhibitor, which interferes with STAT3 transcriptional activity via inhibition of its phosphorylation and nuclear translocation. Bcl-2, Bcl-xL, and Mcl-1, antiapoptotic Bcl-2 family proteins, are highly expressed in human cancer through the enhanced binding of transcription factor STAT3 dimer at their promoter regions. STAT3 homodimer is formed by the phosphorylation of its Tyr705 by the activated Janus-associated kinase (JAK) or Src. Therefore, STAT3 is an intriguing target molecule for cancer treatment by suppressing antiapoptotic Bcl-2 family gene transcription through inhibition of JAK/STAT3/Bcl-2/ Bcl-xL survival pathway employing niclosamide. The drug reverses the radioresistance of human lung cancer to improve the efficiency and efficacy of response in the patients with previous radiotherapy resistance [40].

Since hesperidin regulated various $\mathrm{Bcl}-2$ family protein expressions (Fig. 9), including Bcl-xL (a BH4-domaincontaining Bcl-2 family protein) and Bax, the targeting and/ or co-targeting of these proteins by the small molecules and hesperidin is (are) considered to be a promising strategy for hepatic cancer therapy.

The glycoside forms have commercial potential since it contains sweet taste. The properties of cellular permeability, water solubility, and metabolic change all play pivotal roles in the efficacy of natural phytochemical performance in human beings, which should be concerned to achieve the sufficient amount of active form [41]. The cytotoxic effect against HepG2 cells of naringenin (aglycone) was less than naringin (glycoside form) by MTT assay (Fig. 4). The structural change of the compounds at the molecular level after metabolism or 
digestion affects their functions or bioactivities at cellular level and in animal or human being model. Hesperetin, an aglycone form or an in vivo metabolite of hesperidin, also contains the anticancer and cardiovascular disease prevention and therapy [30].

Although hesperidin is not widely used as a therapeutic drug by itself, it has been in the list of Martindale Extra Pharmacopoeia to be an ingredient of various recipes internationally used for cardiovascular disorders [42]. The roles of hesperidin for treatment of other diseases are in recent clinical trials of applying hesperidin and its derivatives in patients with cardiovascular diseases, including heart failure, atherosclerosis, myocardial infarction, and hypertension. The valuable effects of hesperidin in the treatment of diabetes and dyslipidemia with its antiplatelet and anticoagulant effects are also investigated [30]. Its estrogenic effect, such as decreasing serum and hepatic lipid and reducing osteoporosis in ovariectomized rats, is documented [36]. Hence, hesperidin may be used as a phytoestrogen supplement in menopausal individuals.

The effects of hesperidin on normal cells are reported, such as hesperidin can protect normal renal cells from oxidative injury [43]. It prevents quail neural crest cells from aflatoxin B1 toxicity [44]. Furthermore, the neuroprotective effects of hesperidin in Alzheimer's disease and Parkinson's disease models induced by aluminum chloride [45] and rotenone [46], respectively, are also evidenced. In rats, the chemoprevention of hesperidin in azoxymethane-induced colon carcinogenesis [47] also established. This increases the value of the compound in Citrus by-product, i.e., the seed. Hesperidin has a high potential to be applied for chemoprevention and clinical trials as an anticancer agent.

\section{Conclusion}

Neohesperidin, hesperidin, and naringin are active bioflavonoids found in Citrus seed. Hesperidin induced human hepatocellular carcinoma HepG2 cell apoptosis via both intrinsic and extrinsic pathways. Further, an in vivo model research is required before using hesperidin in the clinical aspects.

Acknowledgments This work was supported by National Research Council of Thailand (NRCT) of the year 2013 and the Commission of Higher Education (CHE) and Thailand Research Fund (TRF) grant no. RMU5080003 for RB; Endowment Research Fund from Faculty of Medicine, Chiang Mai University for PaK, and the Royal Golden Jubilee Ph.D. Program from TRF for WR.

\section{Conflicts of Interest None}

Author contributions RB designed the research, analyzed, and wrote the manuscript. BW carried out the MTT, annexin V/PI, MTP, ROS, caspase assays, and Western blotting of Bax and Bcl-xL. PaK performed the immunoblot of Bid and Bak proteins. WR performed the MTT assay of bioflavonoid and Citrus seed extracts. ND carried out the highperformance liquid chromatograpy technique. PK provided the Citrus seed extract and valuable suggestion. All authors read and approved the final manuscript.

Open Access This article is distributed under the terms of the Creative Commons Attribution 4.0 International License (http:// creativecommons.org/licenses/by/4.0/), which permits unrestricted use, distribution, and reproduction in any medium, provided you give appropriate credit to the original author(s) and the source, provide a link to the Creative Commons license, and indicate if changes were made.

\section{References}

1. Bosch FX, Ribes J, Diaz M, Cleries R. Primary liver cancer: worldwide incidence and trends. Gastroenterology. 2004;127(5 Suppl 1): S5-S16.

2. Jemal A, Bray F, Center MM, Ferlay J, Ward E, Forman D. Global cancer statistics. CA Cancer J Clin. 2011;61(2):69-90.

3. Banjerdpongchai R, Punyati P, Nakrob A, Pompimon W, Kongtawelert P. 4'-Hydroxycinnamaldehyde from Alpinia galanga (Linn.) induces human leukemic cell apoptosis via mitochondrial and endoplasmic reticulum stress pathways. Asian Pac J Cancer Prev. 2011;12(3):593-8.

4. Banjerdpongchai R, Khaw-On P. Terpinen-4-ol induces autophagic and apoptotic cell death in human leukemic HL-60 cells. Asian Pac J Cancer Prev. 2013;14(12):7537-42.

5. Lin W, Tongyi S. Role of Bax/Bcl-2 family members in green tea polyphenol induced necroptosis of p53-deficient Hep3B cells. Tumour Biol. 2014;35(8):8065-75.

6. Galluzzi L, Vitale I, Senovilla L, Eisenberg T, Carmona-Gutierrez $\mathrm{D}$, Vacchelli E, et al. Independent transcriptional reprogramming and apoptosis induction by cisplatin. Cell Cycle. 2012;11(18): 3472-80.

7. Macheix JJ, Fleuriet A, Billot J. Fruit flavonoids: CRC Press, Inc.; 1990.

8. Kim J, Jayaprakasha GK, Uckoo RM, Patil BS. Evaluation of chemopreventive and cytotoxic effect of lemon seed extracts on human breast cancer (MCF-7) cells. Food Chem Toxicol. 2012;50(2):42330 .

9. Tripoli E, Guardia ML, Giammanco S, Majo DD, Giammanco M. Citrus flavonoids: Molecular structure, biological activity and nutritional properties: a review. Food Chem. 2007;104(11):466-79.

10. Nogata Y, Sakamoto K, Shiratsuchi H, Ishii T, Yano M, Ohta H. Flavonoid composition of fruit tissues of citrus species. Biosci Biotechnol Biochem. 2006;70(1):178-92.

11. Perez JL, Jayaprakasha GK, Valdivia V, Munoz D, Dandekar DV, Ahmad $\mathrm{H}$, et al. Limonin methoxylation influences the induction of glutathione S-transferase and quinone reductase. J Agric Food Chem. 2009;57(12):5279-86.

12. Chidambara Murthy KN, Jayaprakasha GK, Kumar V, Rathore KS, Patil BS. Citrus limonin and its glucoside inhibit colon adenocarcinoma cell proliferation through apoptosis. J Agric Food Chem. 2011;59(6):2314-23.

13. Poulose SM, Harris ED, Patil BS. Antiproliferative effects of citrus limonoids against human neuroblastoma and colonic adenocarcinoma cells. Nutr Cancer. 2006;56(1):103-12.

14. Yusof S. G.H. M, Swee KG. Naringin content in local citrus fruits. Food Chem. 1990;37:113-21.

15. Banjerdpongchai R, Kongtawelert P, Khantamat O, Srisomsap C, Chokchaichamnankit D, Subhasitanont P, et al. Mitochondrial and endoplasmic reticulum stress pathways cooperate in zearalenone- 
induced apoptosis of human leukemic cells. J Hematol Oncol. 2010;3:50.

16. Banjerdpongchai R, Suwannachot K, Rattanapanone V, Sripanidkulchai B. Ethanolic rhizome extract from Kaempferia parviflora Wall. ex. Baker induces apoptosis in HL-60 cells. Asian Pac J Cancer Prev. 2008;9(4):595-600.

17. Banjerdpongchai R, Wudtiwai B, Sringarm K. Cytotoxic and apoptotic-inducing effects of purple rice extracts and chemotherapeutic drugs on human cancer cell lines. Asian Pac J Cancer Prev. 2013;14(11):6541-8.

18. Wudtiwai B, Sripanidkulchai B, Kongtawelert P, Banjerdpongchai R. Methoxyflavone derivatives modulate the effect of TRAIL-induced apoptosis in human leukemic cell lines. J Hematol Oncol. 2011;4:52.

19. Gattuso G, Barreca D, Gargiulli C, Leuzzi U, Caristi C. Flavonoid composition of Citrus juices. Molecules. 2007;12(8):1641-73.

20. Iablokov V, Hirota CL, Peplowski MA, Ramachandran R, Mihara $\mathrm{K}$, Hollenberg MD, et al. Proteinase-activated receptor 2 (PAR2) decreases apoptosis in colonic epithelial cells. J Biol Chem. 2014;289(49):34366-77.

21. Estevez S, Marrero MT, Quintana J, Estevez F. Eupatorin-induced cell death in human leukemia cells is dependent on caspases and activates the mitogen-activated protein kinase pathway. PloS One. 2014;9(11):e112536. doi:10.1371/journal.pone.0112536.

22. Banjerdpongchai R, Wudtiwai B, Pompimon W. Stigmalactam from Orophea enterocarpa induces human cancer cell apoptosis via a mitochondrial pathway. Asian Pac J Cancer Prev. 2014;15(23):10397-400.

23. Karthik S, Sankar R, Varunkumar K, Anusha C, Ravikumar V. Blocking NF-kappaB sensitizes non-small cell lung cancer cells to histone deacetylase inhibitor induced extrinsic apoptosis through generation of reactive oxygen species. Biomed Pharmacother. 2015;69:337-44.

24. Lu TH, Su CC, Tang FC, Chen CH, Yen CC, Fang KM, et al. Chloroacetic acid triggers apoptosis in neuronal cells via a reactive oxygen species-induced endoplasmic reticulum stress signaling pathway. Chem Biol Interact. 2015;225:1-12.

25. Smith CC, Guevremont D, Williams JM, Napper RM. Apoptotic cell death and temporal expression of apoptotic proteins bcl-2 and bax in the hippocampus, following binge ethanol in the neonatal rat model. Alcohol Clin Exp Res. 2015;39(1):36-44.

26. Lu Y, Zhang C, Bucheli P, Wei D. Citrus flavonoids in fruit and traditional Chinese medicinal food ingredients in China. Plant Foods Hum Nutr. 2006;61(2):57-65.

27. Saiprasad G, Chitra P, Manikandan R, Sudhandiran G. Hesperidin induces apoptosis and triggers autophagic markers through inhibition of Aurora-A mediated phosphoinositide-3-kinase/Akt/mammalian target of rapamycin and glycogen synthase kinase- 3 beta signalling cascades in experimental colon carcinogenesis. Eur $\mathbf{J}$ Cancer. 2014;50(14):2489-507.

28. Fu Z, Deng B, Liao Y, Shan L, Yin F, Wang Z, et al. The anti-tumor effect of shikonin on osteosarcoma by inducing RIP1 and RIP3 dependent necroptosis. BMC Cancer. 2013;13:580.

29. Shalini S, Dorstyn L, Dawar S, Kumar S. Old, new and emerging functions of caspases. Cell Death Differ. 2015;22(4):526-39.

30. Roohbakhsh A, Parhiz H, Soltani F, Rezaee R, Iranshahi M. Molecular mechanisms behind the biological effects of hesperidin and hesperetin for the prevention of cancer and cardiovascular diseases. Life Sci. 2015;124:64-74.

31. Park HJ, Kim MJ, Ha E, Chung JH. Apoptotic effect of hesperidin through caspase 3 activation in human colon cancer cells, SNU-C4. Phytomedicine. 2008;15(1-2):147-51.
32. Singh DV, Agarwal S, Singh P, Godbole MM, Misra K. Curcumin conjugates induce apoptosis via a mitochondrion dependent pathway in MCF-7 and MDA-MB-231 cell lines. Asian Pac J Cancer Prev. 2013;14(10):5797-804.

33. Ghorbani A, Nazari M, Jeddi-Tehrani M, Zand H. The citrus flavonoid hesperidin induces p53 and inhibits NF-kappaB activation in order to trigger apoptosis in NALM-6 cells: involvement of PPARgamma-dependent mechanism. Eur J Nutr. 2012;51(1):3946.

34. Nazari M, Ghorbani A, Hekmat-Doost A, Jeddi-Tehrani M, Zand $\mathrm{H}$. Inactivation of nuclear factor-kappaB by citrus flavanone hesperidin contributes to apoptosis and chemosensitizing effect in Ramos cells. Eur JPharmacol. 2011;650(2-3):526-33.

35. Rooprai HK, Christidou M, Pilkington GJ. The potential for strategies using micronutrients and heterocyclic drugs to treat invasive gliomas. Acta Neurochir. 2003;145(8):683-90.

36. Meiyanto E, Hermawan A. Anindyajati. Natural products for cancer-targeted therapy: citrus flavonoids as potent chemopreventive agents. Asian Pac J Cancer Prev. 2012;13(2):427-36.

37. Febriansah R, Putri DD. Sarmoko, Nurulita NA, Meiyanto E, Nugroho AE. Hesperidin as a preventive resistance agent in MCF-7 breast cancer cells line resistance to doxorubicin. Asian Pac J Trop Biomed. 2014;4(3):228-33.

38. Han B, Park D, Li R, Xie M, Owonikoko TK, Zhang G, et al. Small-molecule Bcl2 BH4 antagonist for lung cancer therapy. Cancer Cell. 2015;27(6):852-63.

39. Xin M, Li R, Xie M, Park D, Owonikoko TK, Sica GL, et al. Smallmolecule Bax agonists for cancer therapy. Nat Commun. 2014;5: 4935.

40. You S, Li R, Park D, Xie M, Sica GL, Cao Y, et al. Disruption of STAT3 by niclosamide reverses radioresistance of human lung cancer. Mol Cancer Ther. 2014;13(3):606-16.

41. Schwab M, Westermann F, Hero B, Berthold F. Neuroblastoma: biology and molecular and chromosomal pathology. Lancet Oncol. 2003;4(8):472-80.

42. Garg A, Garg S, Zaneveld LJ, Singla AK. Chemistry and pharmacology of the Citrus bioflavonoid hesperidin. Phytother Res. 2001;15(8):655-69.

43. Sahu BD, Kuncha M, Sindhura GJ, Sistla R. Hesperidin attenuates cisplatin-induced acute renal injury by decreasing oxidative stress, inflammation and DNA damage. Phytomedicine. 2013;20(5):45360 .

44. Nones J, Trentin AG. Flavonoid hesperidin protects neural crest cells from death caused by aflatoxin B (1). Cell Biol Int. 2013;37(2):181-6.

45. Thenmozhi AJ, Raja TR, Janakiraman U, Manivasagam T. Neuroprotective effect of hesperidin on aluminium chloride induced alzheimer's disease in wistar rats. Neurochem Res. 2015;40(4):767-76.

46. Tamilselvam K, Braidy N, Manivasagam T, Essa MM, Prasad NR, Karthikeyan S, et al. Neuroprotective effects of hesperidin, a plant flavanone, on rotenone-induced oxidative stress and apoptosis in a cellular model for Parkinson's disease. Oxid Med Cell longev. 2013;2013:102741.

47. Tanaka T, Kohno H, Murakami M, Shimada R, Kagami S, Sumida T, et al. Suppression of azoxymethane-induced colon carcinogenesis in male F344 rats by mandarin juices rich in beta-cryptoxanthin and hesperidin. Int $\mathrm{J}$ Cancer. 2000;88(1): $146-50$. 